\title{
An Advanced APPROACH to Automatic MACHINING OF COMPOSITE PARTS WiTHOUT THEIR Rigid FIXING BY MEANS OF MULTILINK MANIPULATORS WITH STEREO VISION SYSTEM
}

\author{
FILARETOV, V. \& ZUEV, A.
}

Abstract: This chapter presents new advanced approach for automatization of machining of nonrigid composite parts with arbitrary geometrical form. This approach based on using multilink manipulators with stereo vision system and allows not use the expensive individual equipment for rigid fixing parts before processing. This is achieved by high-quality formation and correction of program motions of manipulator cutting tool in real time using information about current position of processed parts from vision system.

Key words: automatic machining, manipulators, vision system, PCM parts
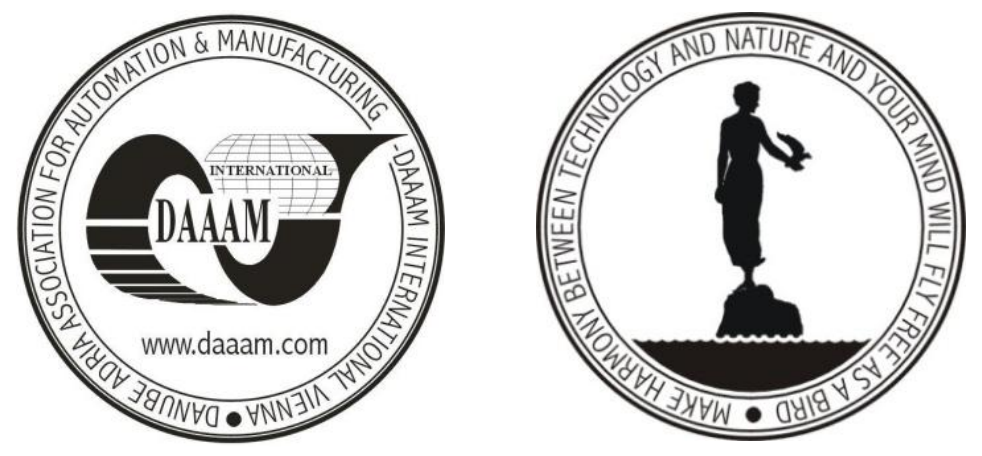

Authors' data: Filaretov, V[ladimir]*; Zuev, A[lexander]**, *Institute of Automation and Process Control, Radio str., 5, Vladivostok, Russia, **Far Eastern Federal University, Sukhanova str., 8, Vladivostok, Russia, filaret@pma.ru, zuev@dvo.ru

This Publication has to be referred as: Filaretov, V[ladimir] \& Zuev, A[lexander] (2013) An Advanced Approach to Automatic Machining of Composite Parts Without Their Rigid Fixing by Means of Multilink Manipulators with Stereo Vision System, Chapter 10 in DAAAM International Scientific Book 2013, pp. 235-256, B. Katalinic \& Z. Tekic (Eds.), Published by DAAAM International, ISBN 978-3-901509-94-0, ISSN 1726-9687, Vienna, Austria

DOI: $10.2507 /$ daaam.scibook.2013.10 
Filaretov, V. \& Zuev, A.: An Advanced Approach to Automatic Machining of Com...

\section{Introduction}

Now the questions of creation and effective implementation into industry (especially into the aircraft industry) of modern mechatronic and robotic systems have big attention. Herewith, one of the most important directions is automatization of technological processes of parts machining from polymer-based composite materials (PCM): cleaning, polishing, cutting, etc. For this purpose, the various automated complexes are widely used. All today known approaches to creation of machining complexes for PCM parts are focused on use of the 5-axial processing centers with numerical programmed control and additional equipment for parts fixing in the course of their machining. In general, this additional equipment is produced individually for each part and has high cost.Conducted researches have shown, that such technology is difficult for realization at producing and machining of PCM parts into of aircraft industry.It is caused by specificity of PCM parts production in aircraft industry, associated with small seriation, but the big nomenclature of produced parts (including large-sized parts).Obviously, that using of individual additional equipment for fixing of each such part is not effective. Besides, outdated technology does not allow to provide the high-speed continuous multicoordinate machining.

Due to noted, it is expediently to change technology of machining of specified PCM parts, having completely excluded use of individual fixing equipment and expensive 5-axial processing centers from technological process, having replaced them with the industrial multilink manipulators (MM) and universal devices of positioning and fixing. The use of MM for automatic processing of PCM parts require ensuring of flexibility of production and convenience of their fast reorganization on machining of new parts. Besides, it is necessary to take into account the main feature of PCM parts production - possible variability of a form of thin-walled spatial parts in the course of their fixing and the subsequent machining. This is inadmissible for the traditional manipulators and processing centers (Craig, 2003). The control program of traditional industrial MM is formed in advance based on computer-aided design models (CAD models) of parts and completely determines program motions of the cutting tool (CT). Any change of the MM parameters and PCM parts (for example at not accurate fixation or at change of its geometrical sizes in the course of forced acting of CT) demands additional reprogramming, use of new procedures of debugging, etc. Noted features require development of essentially new intellectual informational-control system for MM, which will allow in real time to form and correct the operating program of CT motion at machining of spatial PCM parts in the conditions of their possible continuous deformation.

Conducted researches have shown [Fairhurst, 1988; Perks, 2006], that to provide high-quality formation and correction of program CT motions in the course of machining of spatial PCM parts without their rigid fixing in individual equipment it is necessary to use the modern stereo vision systems (SVS) which allow to ensure exact data about processing parts in real time.

This chapter presents results of the researches directed on development of new advanced approach to high-precision automatic machining of spatial PCM parts by means of MM with SVS without their rigid fixing in individual equipment. 


\section{The Analysis of Possible Approaches to Automatic Machining of PCM Parts by Means of MM With SVS}

The conducted preliminary researches show that by means of universal fixing equipment, as a rule, it is possible to fix rigidly in strict accordance with CAD-model only a small set of processed PCM parts. Besides this, the additional deformation of their separate segments at forced milling is possible. The machining of such parts on the basis of data only about CAD models will inevitably lead to errors of motion of their CT and, thereby, - to marriage of machining.

In the course of the analysis of possible approaches to automatic machining of PCM parts by means of MM, three variants of alleged change of their geometrical form were considered (see fig. 1). These variants significantly influence on machining strategy and key technical solutions.

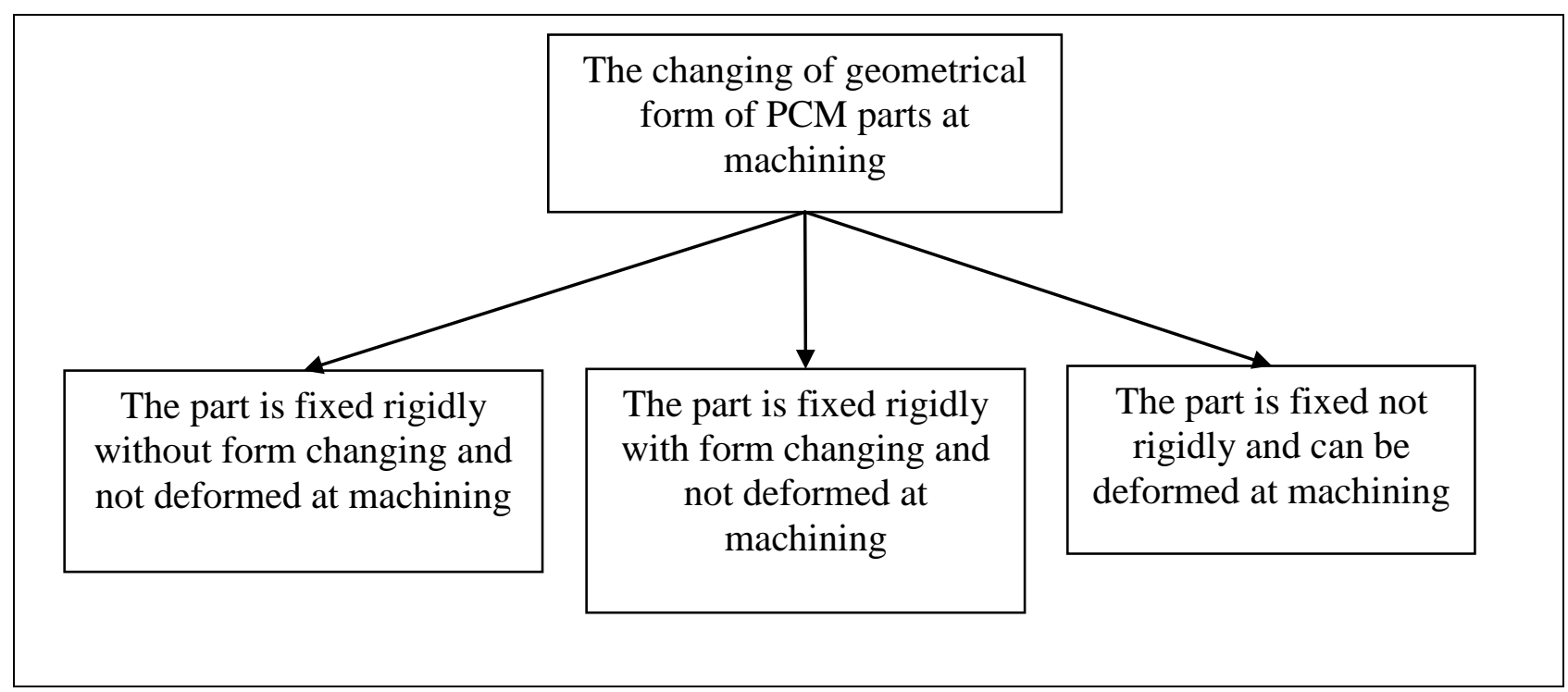

Fig. 1. Possible decisions at automation of PCM parts machining

The first variant assumes that parts are fixed rigidly, correspond to their CAD models and will not deform during machining. This variant is characteristic for not large rigid PCM parts. These parts can be machined by using only CAD-models. Herewith an important task is to ensure fast and exact basing of parts in universal equipment and also definition of them position and orientation in coordinate system of MM. This task can be solved qualitatively with use of modern SVS and optical 3D scanners (Marshall \& Stutz, 2011).

The second variant supposes the rigid fixation of parts in universal equipment without deformation in the course of machining, but not with the corresponding to its CAD - models. In this variant, the important task is formation of geometrical model of spatial CT motion trajectory with taking into account simultaneous movement of positioner with universal equipment on which the processed parts are fixed. This task can be solved also with use of the SVS, which capable to give the exact data about fixed parts. For this purpose, it is necessary to use effective recognition algorithms of cutting line, which are marked on PCM parts (marking of these lines is made in advance after polymerization of composite parts) and creation of program signals for each degree of freedom of MM based on the obtained data. 
The third variant supposes fixation of nonrigid parts with discrepancy of CAD models and their possible deformations in the course of processing. In this variant at automatic formation of CT motion trajectories it is necessary to compensate deformations arising in them from acting of CT. This compensation can be achieved by using information about arising deformations of concrete segments of the marked cutting lines at continuous tracking of their by SVS which are mounted near with end-effector of MM.

Thus, the carried out analysis allows to define three main approaches to automatic machining of PCM parts (see fig. 1): machining by using only CADmodels, machining on in advance marked cutting line by using vision systems and continuous machining by trajectory which are formed in real time. Thus, the first approach is traditional for industrial processing complexes [Madison, 1996]. Further, we will consider features of practical realization of two other described approaches, which are most perspective at realization of modern robotic complexes for machining of spatial PCM parts without their rigid fixation.

\section{Formation of Spatial CT Motion Trajectories for Machining of PCM Parts Which Was Deformed at Fixing in Universal Equipment}

As noted above, the deformation of geometrical form of nonrigid PCM parts at which they any more do not correspond precisely to the CAD models is possible at fixation in universal equipment. Nevertheless, this equipment does not allow the parts deformation at the subsequent force impact of CT on their surface in the course of spatial machining.

At automatic machining of such parts by means of MM on their surface according to $\mathrm{CAD}$ models by known way cutting lines are marked, which at mechanical fixing of details also are deformed. Therefore, before machining of already fixed parts it is necessary to provide the automatic formation of the current position of earlier marked spatial cutting lines by using SVS. However, on average and large-size PCM parts of difficult form, being in an initial position, SVS can identify only one segment of marked line. For formation of full spatial model of cutting line in system of coordinates (SC) connected with the basis of MM, it is necessary to provide the corresponding movements of SVS or equipment on which processed parts are fixed. This procedure will be described below.

In paper [Sebastian et all, 2009] the problem of creation of models of large-size objects by using mobile television cameras is solved. However, the offered algorithms allow to receive spatial coordinates of separate segments of considered objects only at the small curvature of their recognizable contours. In papers [Klank et all, 2009 \& Le Duc, 2012] for the solution of a problem of movement of MM from one object of working area to another, the methods of formation of the SVS movements fixed on end-effector are offered. As showed researches, these methods do not allow to form qualitatively program movements of MM based on SVS when tracking not completely visible contours.

As a whole, the made analysis showed that when SVS is moving in space (round the object) by using mobile basis, the essential errors in determination of spatial coordinates of observed objects arise (Gruen\& Huang, 2001). For elimination of this problem, it is necessary to use the high precision and expensive systems for 
SVS motion. But more effectively instead SVS motion to use the rather cheap serial two-axial positioners allowing precisely to turn parts fixed on them in vertical and horizontal axes (i.e. move the parts).

In works (Mezouar\&Chaumette, 2002; Comport et all, 2006), the approach allowing to consider the partial objects movement in STZ view is offered, but the problem of formation of program signals of position and orientation image changing for obtaining demanded information about processed object isn't considered.

Important problem at creation of full and exact spatial models of cutting lines marked on large-size parts at changing of their spatial position is "stitching" of coordinates of points of received segments of lines by means of SVS to uniform spatial line. For the solution of this task there is some approaches, however all of them are focused on use of difficult system of the calibration templates which application in the conditions of real production is ineffective.

Thus, the task of development of creation method of models of spatial cutting lines for large-size parts with difficult form by means of SVS and two-axial positioner clamp of PCM parts is set. This method should ensure continuous by-turn getting in SVS field of view of all segments of extensive marked cutting lines and subsequent stitching of these segments.

At the solution of this task, we will use the generalized block diagram of the robotic machining complex shown in Fig. 2. This complex consists of MM 1, SVS 2, two-axial positioner 3 on which by means of universal fixing equipment the nonrigid PCM part 4 with the marked cutting line 5 is fixed.

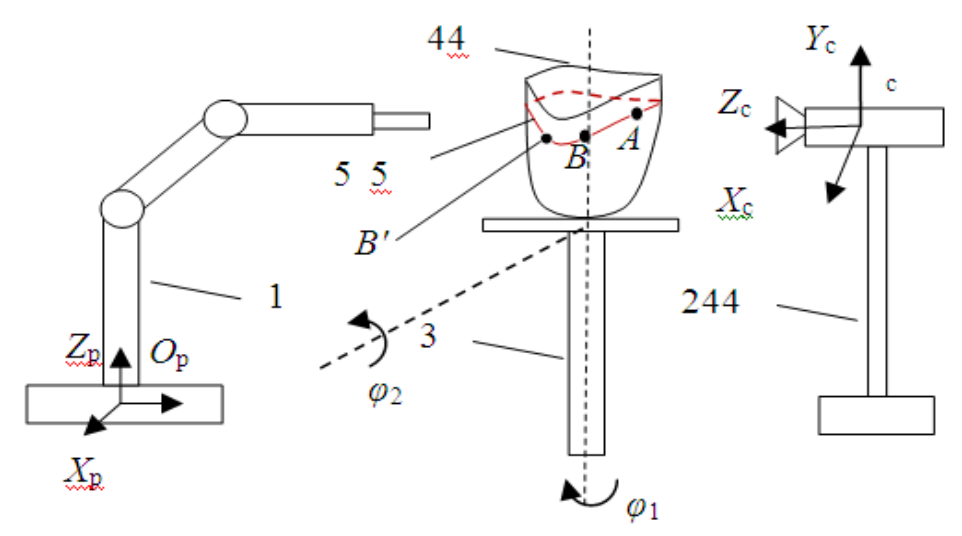

Fig. 2. Generalized block diagram of the robotic machining complex

Initially, only small segment $\mathrm{AB}$ of marked cutting line on large-size parts with difficult form may be seen at firmly fixed in the space SVS. After identification of this segment (based on known algorithms (Forsyth \& Ponce, 2011; Shapiro, 2001) and construction of its spatial model, it is necessary to turn this part by means of positioner-fixator so that the next segment of line would be seen. At that, for stitching all segments of cutting line, it is necessary to ensure movement of point $A$ to point $B$, and point $B$ to other frontier point of the next segment of cutting line in tangent plane of SVS and so forth. In order to solve this task, it is proposed to use the following three-stage algorithm. 
Filaretov, V. \& Zuev, A.: An Advanced Approach to Automatic Machining of Com...

Before the first stage, for the correct calibration of SVS it is necessary to define the position and orientation of the camera in SC of MM. For this purpose it is offered to use a method (Grammatikopouloset all, 2004; ZuWhan, 2006), based on fixing arbitrarily on surface of part of special rectangular tag. This method is based on the analysis of so-called "disappearing points" (ZuWhan, 2006), formed by crossing in the picture planes of SVS the distorted parties of this rectangle and allows to receive required vector of position of part $T^{k} \in R^{3}(k=1)$ and a matrix of its orientation $R^{k} \in R^{3 \times 3}(k=1)$ is relative to coordinate system connected with the basis of MM. These a matrix and a vector look like:

$$
\begin{aligned}
R^{k} & =\left[\begin{array}{llll}
v_{x} & v_{y} & v_{x} & \times v_{y}
\end{array}\right], \\
T^{k} & =\left[\begin{array}{l}
X_{\mathrm{p}_{s}} \\
Y_{\mathrm{p}_{s}} \\
Z_{\mathrm{p}_{s}}
\end{array}\right]-R^{k}\left[\begin{array}{c}
X_{\mathrm{cs}} \\
Y_{\mathrm{cs}} \\
Z_{\mathrm{cs}}
\end{array}\right],
\end{aligned}
$$

where $v_{x}, v_{y} \in R^{3}$ are vectors of coordinates of two disappearing points in SC of SVSO $O_{\mathrm{c}} X_{\mathrm{c}} Y_{\mathrm{c}} Z_{\mathrm{c}} ;\left[X_{\mathrm{cs}}, Y_{\mathrm{cs}}, Z_{\mathrm{cs}}\right]^{\mathrm{T}}$ is a vector of coordinates of any top of a rectangle in $\mathrm{SC} O_{\mathrm{c}} X_{\mathrm{c}} Y_{\mathrm{c}} Z_{\mathrm{c}} ;\left[X_{\mathrm{ps}}, Y_{\mathrm{ps}}, Z_{\mathrm{ps}}\right]^{\mathrm{T}}$ is a vector of coordinates of this top of the specified rectangle in $\mathrm{SC}$ of the basis of MM of $O_{\mathrm{p}} X_{\mathrm{p}} Y_{\mathrm{p}} Z_{\mathrm{p}}$.

At the first stage by means of methods of recognition of images (Forsyth \& Ponce, 2011), spatial coordinates of boundary points of visible SVS of a segment of marked line in $\mathrm{SCO}_{\mathrm{c}}, X_{\mathrm{c}}, Y_{\mathrm{c}}, Z_{\mathrm{c}}$ are obtained.Herewith (see fig. 3) for the subsequent planning of a positioner motions it is necessary to consider not all visible segment $A B$, but only its part - segment $A D$, where $D_{1}=\left[\begin{array}{ll}U_{\mathrm{t}_{i} \mathrm{~B}}-\mu_{U} & V_{\mathrm{t}_{i} \mathrm{~B}}+\mu_{V} \\ 1\end{array}\right]^{T}$ is a uniform coordinates of point $D_{l}$ in $i$-oh pixel's CS of SVS. This procedure repeats for all segments of line for full viewing of all this marked line. Introduction of point $D_{l}$ is necessary for high-quality "sewing together" of all segments of marked line after the next turn of positioner as at a difficult type of this line losses of extreme points of considered segments are possible.

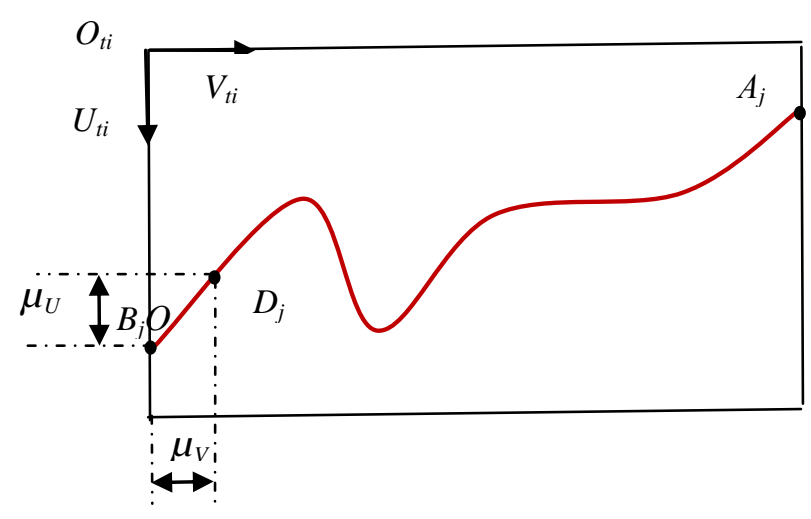

Fig. 3. The image $j$-th segment of marked cutting line concerning to pixel SCO $O_{t i} U_{t i} V_{t i}$ of the picture plane of SVS 
In fig. 3 the following designations are entered: $j$ is a number of the next segments of cutting line $(j=1,2,3, \ldots) ; \mu_{U}, \mu_{V}$ are parameters of shift of a point $D_{j}$, chosen at the SVS control, $i=1,2$ is number of pixel SC of SVS.

At the second stage, considering already received spatial arrangement of the next segment $A_{j} D_{j}$ it is possible to define desiredjoints rotation of positioner $\varphi_{1}{ }^{d}$, $\varphi_{2}{ }^{d}$ for combination of boundary points of $A_{j}$ and $D_{j}$ (for combination of straight lines the $O_{\mathrm{c}} A$ and $\left.O_{\mathrm{c}} D\right)$. For the explanation of the solution of this task it is possible to use fig. 4. Apparently from fig. 4, for combination of direct $O_{\mathrm{c}} A$ and $O_{\mathrm{c}} B$ it is necessary to execute two turns of a piece of $O_{\mathrm{c}} A$ : round the $O_{\mathrm{c}} Y_{\mathrm{c}}$ axis on a corner $\varphi_{1}^{*}$ and then round an $O_{\mathrm{c}} X_{\mathrm{c}}$ axis on a corner $\varphi_{2}^{*}=\beta_{D}-\beta_{A}$ which are calculated by means of expressions

$$
\begin{aligned}
& \varphi_{1}^{*}=\alpha_{A}+\alpha_{D}=\operatorname{arctg}\left(\frac{X_{c}^{D}}{Z_{c}^{D}}\right)+\operatorname{arctg}\left(\frac{X_{c}^{A}}{Z_{c}^{A}}\right), \\
& \varphi_{2}^{*}=\beta_{D}-\beta_{A}=\operatorname{arctg}\left(\frac{Y_{c}^{D}}{\sqrt{X_{c}^{D}+Z_{c}^{D}}}\right)-\operatorname{arctg}\left(\frac{Y_{c}^{A}}{\sqrt{X_{c}^{A}+Z_{c}^{A}}}\right) .
\end{aligned}
$$

Considering that $\varphi_{1}^{*}, \varphi_{2}^{*}$, define SVS turn for combination of noted straight lines, upon transition to turns of axes of a positioner in expressions (1) it is necessary to change signs, that is

$$
\begin{aligned}
& \varphi_{1}^{d}=-\operatorname{arctg}\left(\frac{X_{c}^{D}}{Z_{c}^{D}}\right)-\operatorname{arctg}\left(\frac{X_{c}^{A}}{Z_{c}^{A}}\right), \\
& \varphi_{2}^{d}=-\operatorname{arctg}\left(\frac{Y_{c}^{D}}{\sqrt{X_{c}^{D}+Z_{c}^{D}}}\right)+\operatorname{arctg}\left(\frac{Y_{c}^{A}}{\sqrt{X_{c}^{A}+Z_{c}^{A}}}\right) .
\end{aligned}
$$

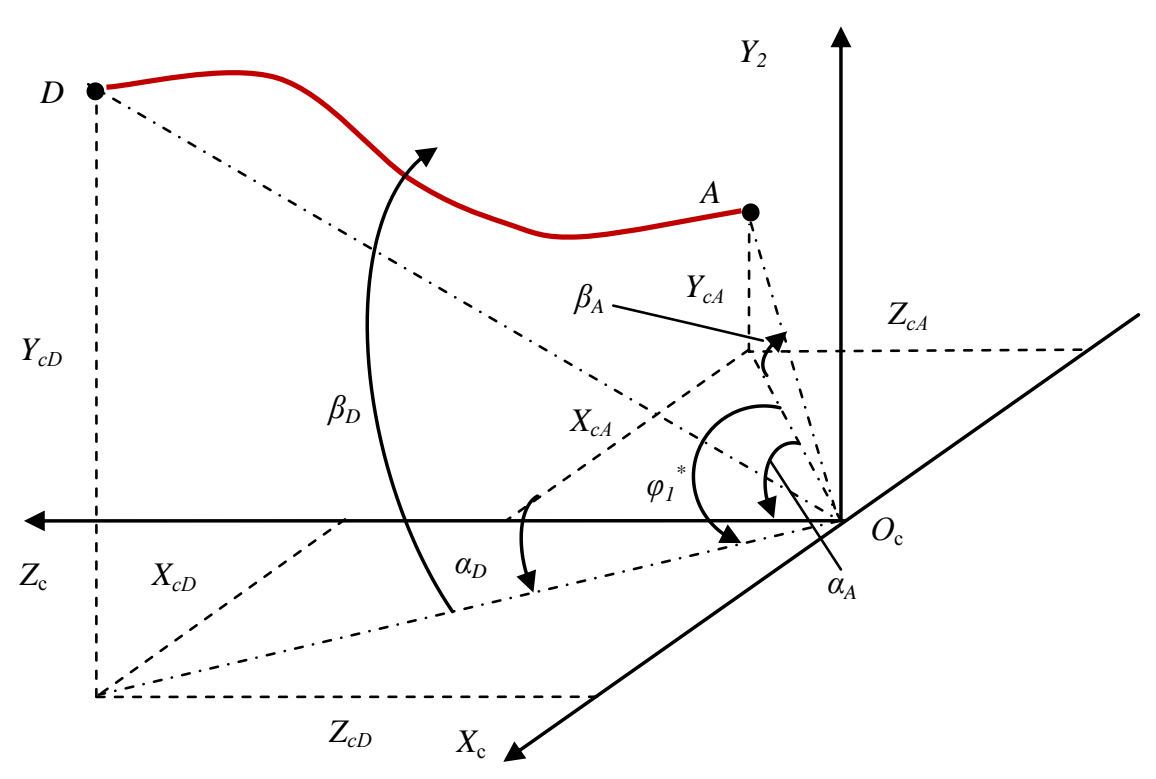

Fig. 4. Mutual location of points $A$ and $D$ of cuttingline in $\mathrm{SC} O_{\mathrm{c}} X_{\mathrm{c}} Y_{\mathrm{c}} Z_{\mathrm{c}}$ 
Filaretov, V. \& Zuev, A.: An Advanced Approach to Automatic Machining of Com...

Using the equations (2) for formation of program signals of positioner motion, when machining of large-size PCM parts it is possible to joint all segments of marked cutting lines.

At the third stage of algorithm for correct "stitching" of boundary points of all segments of cutting line in a uniform contour when moving PCM part by positioner it is necessary to redefine constantly coordinates stated above a vector $T^{j}$ and a matrix $R^{j}$ taking into account this movement as follows $(j=1,2,3, \ldots)$ :

$$
\begin{gathered}
R^{k+1}=\left[\begin{array}{ccc}
1 & 0 & 0 \\
0 & \cos \varphi_{2} & -\sin \varphi_{2} \\
0 & \sin \varphi_{2} & \cos \varphi_{2}
\end{array}\right]\left[\begin{array}{ccc}
\cos \varphi_{1} & 0 & \sin \varphi_{1} \\
0 & 1 & 0 \\
-\sin \varphi_{1} & 0 & \cos \varphi_{1}
\end{array}\right] R^{k}, \\
T^{k+1}=\left[\begin{array}{c}
X_{\mathrm{p}_{s}} \\
Y_{\mathrm{p}_{s}} \\
Z_{\mathrm{p}_{s}}
\end{array}\right]-R^{k+1}\left[\begin{array}{c}
X_{\mathrm{cs}} \\
Y_{\mathrm{cs}} \\
Z_{\mathrm{cs}}
\end{array}\right] .
\end{gathered}
$$

Besides, at the third stage it is necessary to define the moment of completion of work of the described algorithm when SVS will determine put on a processed part all necessary coordinates of an extended spatial cutting line. It is possible to realize by using indications of the sensor of joint $\varphi 1$. Work of algorithm stops when the value of $\varphi_{1}$ will be equal or exceeds to 360 degree.

Thus, the considered algorithm allows to form automatically program signals of movement of a two-axial positioner with universal fixation equipment of PCM parts, providing obtaining all necessary coordinates of an extended spatial cutting lines.

For high quality, machining of PCM parts besides exact tracking of program cutting line it is necessary to provide also the set orientation of CT to surface of these parts. As a rule, it is required to provide CT arrangement by normal to cutting line (Youdong, 2000; Puppo, 1998). Thus, after allocation of the points forming cutting line using (2), and obtaining their spatial coordinates it is necessary to solve a problem of calculation of normal vector to surface of parts in all points of this line.

As showed the conducted researches, there are two approaches to calculation of normal vector to a surface of machined parts for points of cutting line of distinguished by means of SVS. Application them depends on parameters of possible PCM parts deformations. If by means of universal fixing equipment it is possible to fix PCM parts without deformation or with rather small deformation of its separate segments, it is possible to use the approach based on obtaining information on normal vectors to surfaces from CAD-models of these parts. For realization of this approach, it is necessary to combine correctly points of marked line really located in PCM part with the corresponding points of cutting trajectory on CAD-model. After that, it is possible to use already known normal vectors to the corresponding combined points, available in CAD models of the corresponding parts. If at parts fixation, there are essential deformations of its some processed segments, it is necessary to use the approach based on use of optical 3D scanners, allowing to receive exact 3D models of machined PCM parts. 
The coordinates of PCM parts surfaces points (received by means of the 3D scanner) located on both sides from cutting line allow to define the required normal vectors with use of primitives in the form of triangles. Thus for each point of line two triangles which have to be located are under construction so that one of their tops was on cutting line and two others in some small vicinity from one and other its party (see fig. 5).

Thus, knowing spatial coordinates of the received tops of triangle in SC connected with the basis of $\mathrm{MM}$, it is possible to calculate all necessary normal vectors to points of cutting line, really located on parts, by means of the following expression (expressions for calculation of normal vector to surface in point A in fig. 5 are shown):

$$
\begin{gathered}
N_{A}=\frac{N_{A}^{1}+N_{A}^{2}}{2}, \\
N_{A}^{1}=\left[X_{\mathrm{p} B}-X_{\mathrm{p} A}, Y_{\mathrm{p} B}-Y_{\mathrm{pA}}, Z_{\mathrm{p} B}-Z_{\mathrm{pA} A}\right] \times\left[X_{\mathrm{pC}}-X_{\mathrm{pA}}, Y_{\mathrm{pC}}-Y_{\mathrm{pA}}, Z_{\mathrm{pC}}-Z_{\mathrm{p} A}\right], \\
N_{A}^{2}=\left[X_{\mathrm{p} E}-X_{\mathrm{p} A}, Y_{\mathrm{p} E}-Y_{\mathrm{p} A}, Z_{\mathrm{p} E}-Z_{\mathrm{p} A}\right] \times\left[X_{\mathrm{p} D}-X_{\mathrm{pA} A}, Y_{\mathrm{p} D}-Y_{\mathrm{pA}}, Z_{\mathrm{p} D}-Z_{\mathrm{pA}}\right],
\end{gathered}
$$

where $X_{\mathrm{pk}}, Y_{\mathrm{p} k}, Z_{\mathrm{p} k}$ is coordinate of $k$-th point in $\mathrm{SC}$ of $\mathrm{MM}(k=A, B, C, D, E, \ldots)$.

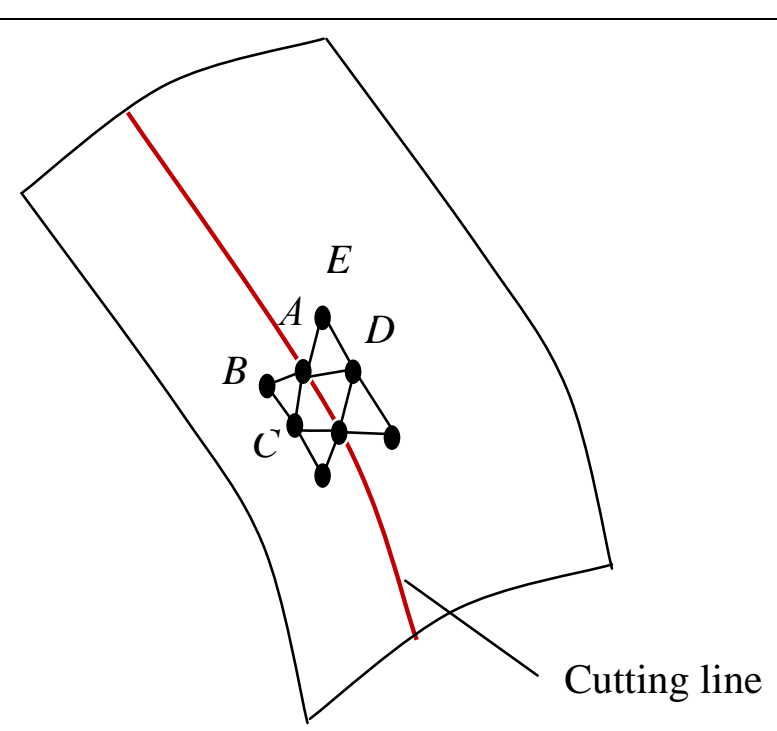

Fig. 5. Approximation of a surface of a detail by triangles in the field of cutting line

It is important to note that length of the parties of the triangles used for approximation of surfaces, has to get out depending on curvature of these surfaces: the more their curvature in vicinity of cutting line, the there have to be sizes of triangles less, reducing thus the accuracy of defined normal vectors.

Thus, the considered two approaches allow to define normal vectors to PCM parts surfaces on which pass cutting lines by existing SVS. As a whole it is possible to note that the considered algorithms allow to carry out high-quality processing of various PCM parts in case of their rigid fixing with deformation of geometrical form. 
Filaretov, V. \& Zuev, A.: An Advanced Approach to Automatic Machining of Com...

\section{Machining of PCM Parts Without Rigid Fixing at Presence of Deformation Deviation of Form From Mathematical CAD - Models}

As it was noted above, deformations of geometrical form of PCM parts at the fixing in universal equipment can occur. The specified equipment also permit deformation of the parts fixed in it at the subsequent force acting of CT on their surface in the course of spatial machining. As a rule, such deformation is possible at milling of thin-walled PCM parts (Filaretov et all, 2011).

For machining of such parts it is necessary to form and correct automatically in the course of processing the CT motion trajectory on the basis of information on the current arrangement of the parts of trajectories of cutting marked on surfaces, received by means of SVS. Thus the SVS has to be fixed on end-effector of MM near with CT (Chaumette, \& Hutchinson, 2006 and 2007). The generalized block diagram of the robotic complex constructed on the basis of this principle shown on fig. 6 .

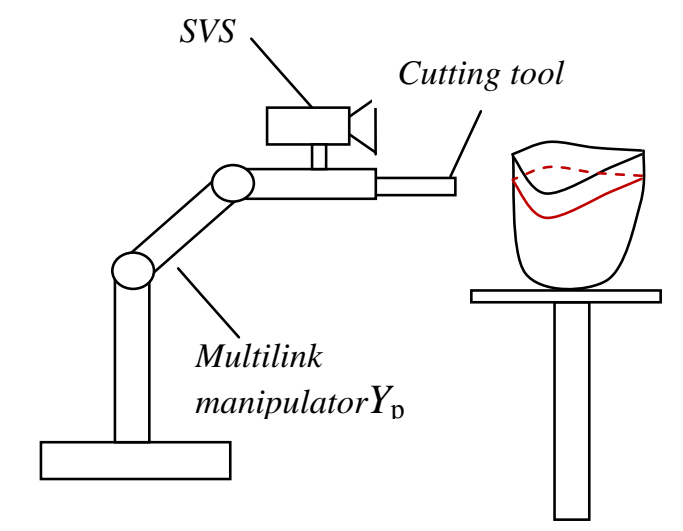

Fig. 6. The generalized block diagram of robotic complex for machining at the presence of deformation of PCM parts

At the machining of deformable details it is necessary to determine previously coordinates of all points of the marked trajectory of cutting by the approach developed in the previous section. Further the MM begins cutting of parts in real time on the recognizable trajectory of cutting. Thus the SVS fixed on end-effector, carries out shooting of a site of a trajectory before CT and in case of its deformation of control system of $\mathrm{MM}$ carries out correction of originally certain trajectory of movement CT by means of the data received from SVS. Let's consider the method of implementation of this correction.

It is possible to determine two components of arising deformation of processed segment of PCM part: lengthwise and crosscut deformations. The analysis showed that deformation of PCM parts at the milling caused by lengthwise acting of CT will be inconsiderable. The main contribution to deformation of processed PCM parts will be made by crosscut influence of CT, therefore further the main attention will be paid to research of crosscut deformation.

One of the possible advanced approaches for machining PCM parts with arising deformation is to use the automatic formation and correction of CT motion 
trajectories with use of information on current state of processed segments of these parts. At realization of this approach it is supposed that program values of coordinates of the points forming cutting line of fixed parts are stored in memory of control system of MM. These coordinates are formed in advance in coordinate system connected with the basis of MM and can be received using CAD models of details or by means of methods of recognition marked cutting line.

Except coordinates of the points cutting trajectory in computer memory also admissible values of crosscut deformations of current segments $\delta^{d}{ }_{l}(l=1, \ldots, N$, where $N$ is a quantity of set points of cutting line) in $i$-th trajectory points are setted. These admissible values, generally, can be various for each of sites of processed parts and are defined during experimental studies for each concrete PCM part. On the basis of the obtained in advance and current data of control system of MM can form program speeds of movement CT on concrete cutting trajectories.

Process of milling of PCM part (see fig. 7) begins with a moving of CT to its surface in some initial point of cutting line (see $A_{1}$ point in fig. 7) according to its CAD model. Thus at cutting down of mill the deformation of not rigidly fixed segment of part can begin, and at further movement of CT to next point of trajectory $A_{2}$ (in advance created on the basis of CAD-model) there will be a mill descent from a trajectory of cutting. It will lead to a stop of process of milling and by the beginning of new procedure of the CT installation on this trajectory.

To eliminate the arising deformation it is necessary to introduce the corresponding corrections in originally set cutting trajectory using SVS near with CT. This SVS defining the current position of CT concerning to cutting line should provide the calculation of real spatial coordinate of the following point of cutting line on deformable segment of PCM part (see $A_{2}^{*}$ point in fig. 7). At this the control system of MM in tracing mode has to remove an arising mismatch between program coordinates of trajectory points and coordinates of real points, continuously received by means of SVS. Herein it's necessary to consider also continuously the size of the current deformation of parts in new real point. If arising deformation $\delta_{l}$ exceeds admissible $\delta_{l}^{d}$ on a considered segment of part, it is necessary to reduce the speed of movement of CT in the specified direction until the condition $\delta_{l}<\delta^{d}{ }_{l}$.will start being satisfied.

For realization of automatic system of adaptive fine tuning of speed of movement of CT on the basis of the size of arising current deformation of PCM part it is possible to use the approaches described in works (Filaretov et all, 2012).

Another approach to working off of arising deformations is to use of force/position control system for MM providing not only exact movement of CT on the set trajectory, but also simultaneous control of its force impact on PCM part surface.As showed the conducted researches for development of high-precision force/position control system at the solution of problems of milling of nonrigid PCM parts it is possible to use simultaneous force/position control system of MM (Zuev\&Filaretov, 2009) which without use of force sensors can provide high-quality performance of operations on machining of difficult PCM parts (including with high speed). 


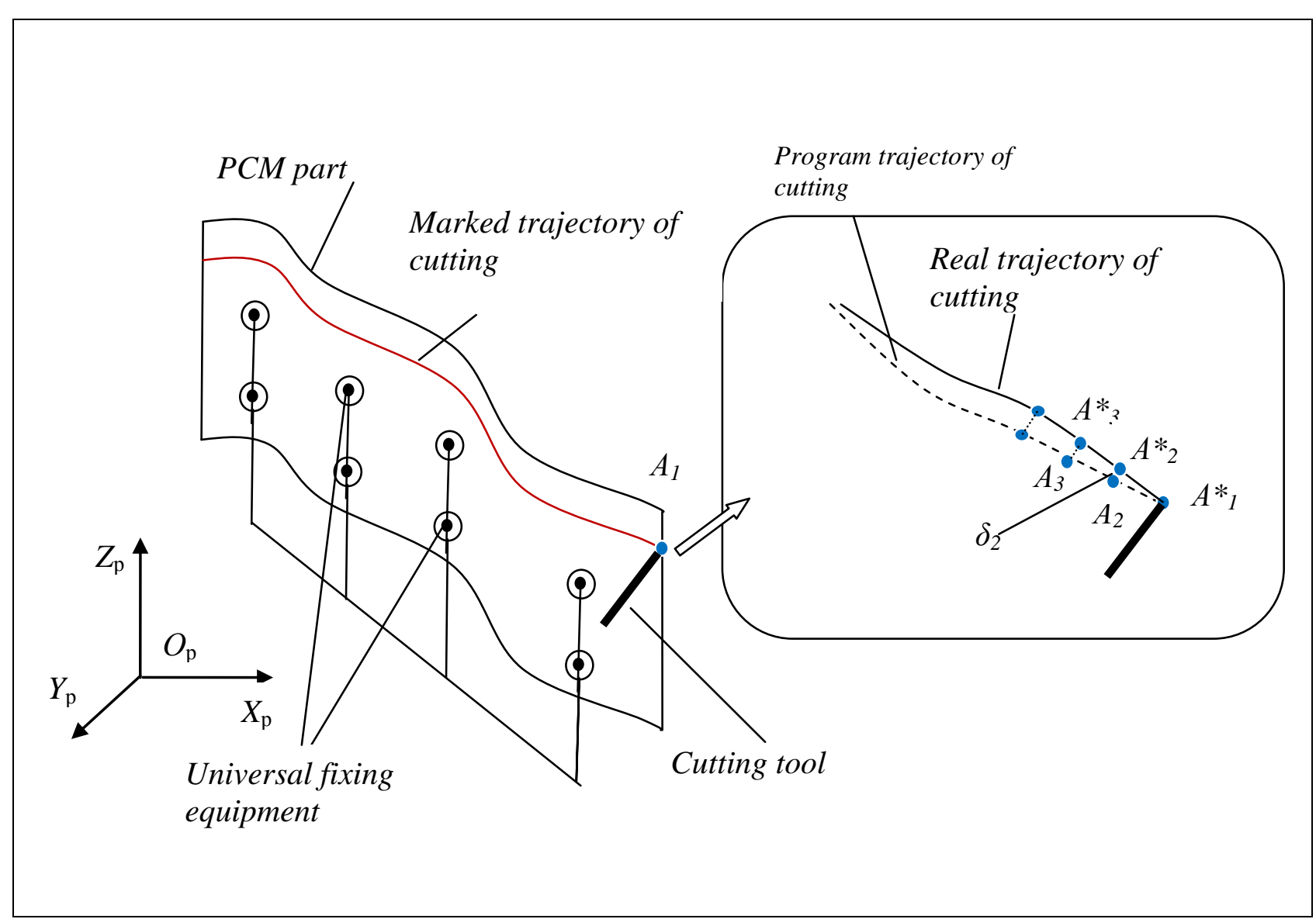

Fig. 7. Illustration of deformation of processed details under the influence of CT

For effective realization of synthesized control system in real time it is necessary to form not only algorithm of creation of program trajectories of spatial movement CT with demanded orientation, but also the desirable law of change of the external moments developed by all MM electric drives for control of demanded force impact of this tool on a surface of the processed composite part. Let's consider the features of development such force-position control system for industrial MM in more detail.

The simultaneous force/position control systems should be synthesized at the level of the servo actuators of manipulators because the dynamic properties of robot are formed in this level. Therefore using known principle of decomposition the control system of manipulator is divided off to the separate control subsystems of actuators, however, the interactions between all degrees-of-freedom (DOF) of manipulator should be taken into account and completely preserved for designing the qualitative control. In this case each actuator of manipulator must trace own trajectory of motion and create moment necessary for the desired spatial motion of $\mathrm{CT}$ and for obtaining the given force.

The synthesis of force/position regulator for each actuator allowing to control the desired rotation angle of output shaft and simultaneously to create the desired external moment (for force creation) is performed with the help of the optimal controller analytical design method with the quadratic functional. Due to the special selection of the phase coordinates of actuators and their desired values the position errors and moment errors will be simultaneously minimized. We have developed a 
special method for definition of desirable external moments necessary for creation of the force. However traditional optimal controller analytical design method requires the presence of the linear objects of control with constant parameters but each actuator of the moving manipulator is nonlinear and has substantially variable parameters because of interaction and viscous frictions. Therefore for the successful application of this method it is necessary to stabilize parameters of each actuator at some nominal level and thus provide invariance to variable parameters and elimination of interaction.

Thus the following synthesis sequence of simultaneous force/position control is proposed. At the first step we will synthesize the adaptive regulators of inner contour for each actuator which compensate the interactions between all DOF and allow to describe these actuators by the linear differential equations with constant coefficients. At the second step for each actuator with the already stabilized nominal parameters the force/position regulators will be synthesised. Finally the problem of determining such values of the desired external moments for each actuators which provide required force will be solved.

Synthesis of simultaneousforce/position control system we will solve on an example of 6 DOFindustrial MM represented on fig. 8.

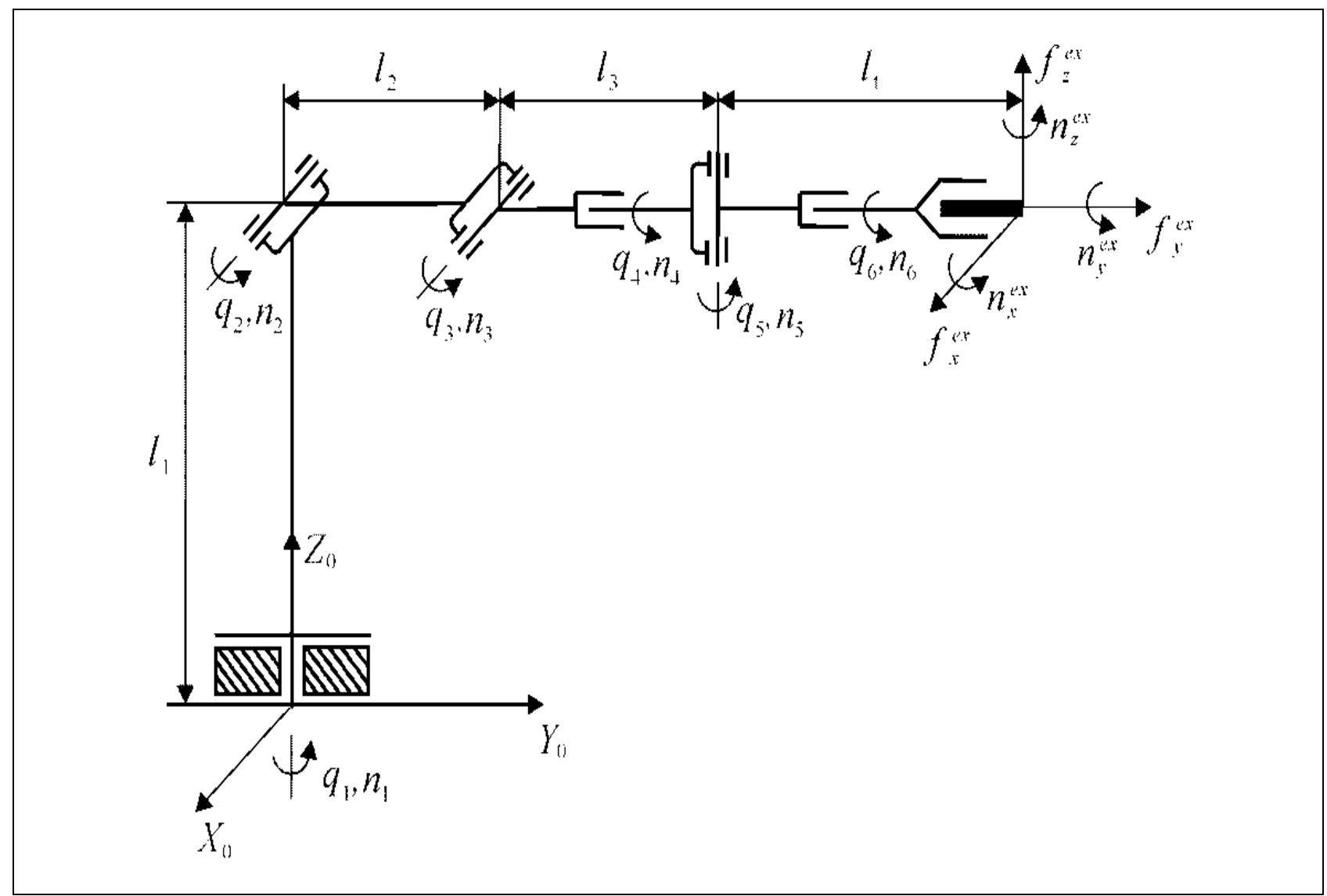

Fig. 8. Kinematical scheme of the MM ( $L_{i}$ is length of $i$-th link; $L_{t}$ is length of CT)

It is expediently to use the second form Lagrange equation to obtain the moment torques $P_{i}$ in each DOF of the MM (see Fig. 8) while moving its CT with force 
Filaretov, V. \& Zuev, A.: An Advanced Approach to Automatic Machining of Com...

exerted by it if laws of change manipulator's generalized coordinates $q_{i}(i=\overline{1,6})$ are known.

The expressions for moments acting in each DOF of manipulator can be represented in the form

$$
P_{i}=H_{i}(q) \ddot{q}_{i}+h_{i}(q, \dot{q}) \dot{q}_{i}+n_{i}(q)+M_{E i}(q, \dot{q}, \ddot{q}),
$$

where $H_{i}$ is diagonal element of matrix of inertia of manipulator; $h_{i}$ is element of the vector of coriolis and centrifugal forces; $n_{i}$ is external moment (part of $P_{i}$ ) developed by $i$-th actuator which in aggregate with the similar moments of other actuators provides force from CT to PCM part; $M_{E i}$ is moment which represents interactions acting to $i$-thDOF from other manipulator links and also action of the gravitational forces. Values $H_{i}, h_{i}$ and $M_{E i}$ with the help of second Lagrange equation are defined.

Dynamics representation in such form (3) rather then in recursive form (method Newton-Euler) conveniently for us in the further synthesis of an adaptive regulator because therein the analytical representation $H_{i}, h_{i}$ and $M_{E i}$ is necessary.

The equations of electric and mechanical chains of the MM actuators in view of expression (3) have the following form

$$
\begin{aligned}
& L_{i} \frac{d I_{i}}{d t}+R_{i} I_{i}+K_{E i} \dot{\alpha}_{i}=K_{A i} U_{i}^{*}, \\
& M_{M i}=\left(H_{i}^{*}+J_{i}\right) \ddot{\alpha}_{i}+\left(h_{i}^{*}+K_{V F i}\right) \dot{\alpha}_{i}+n_{i}^{*}+M_{E i}^{*}+M_{Q F i}=K_{M i} I_{i},
\end{aligned}
$$

where $R_{i}, L_{i}, I_{i}$ are resistance, inductance and current of electric motor rotor circuits, accordingly; $K_{E i}$ is counter-EMF coefficient; $K_{M i}$ is moment coefficient; $\alpha_{i}$ is turn angle of electric motor shaft; $K_{A i}$ is amplifier coefficients; $U_{i}^{*}$ is input voltage of amplifiers; $M_{M i}$ is moment provided by electric motor; $J_{i}$ is moment of inertia of electric motor shaft and rotating parts of reducers; $H_{i}^{*}, h_{i}^{*}, M_{E i}^{*}, n_{\mathrm{i}}^{*}$ are respective values determined by the equations $H_{i}^{*}=\frac{H_{i}}{i_{R i}^{2}}, h_{i}^{*}=\frac{h_{i}}{i_{R i}^{2}}, M_{E i}^{*}=\frac{M_{E i}}{i_{R i}}, n_{\mathrm{i}}^{*}=\frac{n_{i}}{i_{R i}} ; K_{V F i}$ is viscous friction coefficient; $M_{Q F i}$ is Columbus friction moment; $i_{R i}$ is reducing ratio of the reducer; $i$ is a number DOFof the MM.

The differential equation of loaded electric servo actuator, controlling the angle of $i$-th manipulator's DOF can be expressed as

$$
\begin{aligned}
& L_{i}\left(H_{i}^{*}+J_{i}\right) \dddot{\alpha}_{i}+\left[L_{i}\left(2 h_{i}^{*}+K_{V F i}\right)+R_{i}\left(H_{i}^{*}+J_{i}\right)\right] \ddot{\alpha}_{i}+\left[L_{i} \dot{h}_{i}^{*}+R_{i}\left(h_{i}^{*}+K_{V F i}\right)+K_{E i} K_{M i}\right] \dot{\alpha}_{i}+ \\
& +R_{i}\left(n_{\mathrm{i}}^{*}+M_{E i}^{*}+M_{Q F i}\right)+L_{i}\left(\dot{n}_{\mathrm{i}}^{*}+\dot{M}_{E i}^{*}\right)=K_{A i} K_{M i} U_{i}^{*},
\end{aligned}
$$

where $\dot{h}_{i}^{*}=\frac{\dot{h}_{i}}{i_{R i}^{2}}$ and $\dot{M}_{E i}^{*}=\frac{\dot{M}_{E i}}{i_{R i}}$ are reduced to motor shaft values $\dot{h}_{i}$ and $\dot{M}_{E i}$, which on the basis of an analytical expressions for $H_{i}, h_{i}$ and $M_{E i}$ (see (3)) are determined. 
The equation (5) has significantly changing coefficients because of sufficient changing in parameters $H_{i}^{*}, h_{i}^{*}, \dot{h}_{i}^{*}, M_{E i}^{*}$ and $\dot{M}_{E i}^{*}$. Hence electric servo actuators described by equation (5) have changing dynamic properties and changing quality parameters of their work.

To stabilize dynamic properties and quality parameters of servo actuators described by differential equation (5) at some nominal level the approach, proposed in (Filaretov, 1993; Filaretov\&Vukobratovic, 1995) can be used. Let the differential equation describing the dynamics of the servo actuator with constant nominal parameters and stable dynamic properties at the force/position control be

$$
L_{i}\left(J_{n i}+\dot{n}_{i}^{*}\right) \dddot{\alpha}_{i}+R_{i}\left(J_{n i}+n_{i}^{*}\right) \ddot{\alpha}_{i}+K_{E i} K_{M i} \dot{\alpha}_{i}=K_{A i} K_{M i} U_{i},
$$

where $J_{n i}$ is nominal value of moment of inertia; $U_{i}$ is control signal, coming to the input of the correction system.

To obtain the law of the self-tuning, which provides the transformation of the equation (5) to the equation (6), first to be done is to express the highest order derivative and substitute it to the equation (5). After the substitution for each electric servo actuator of the manipulator we can obtain the signal $U_{i}^{*}$, which provides the transformation of equation (5) with sufficiently changing parameters, to equation (6) with constant nominal parameters

$$
\begin{aligned}
& U_{i}^{*}=\left(\left(J_{i}+H_{i}^{*}\right) / J_{n i}\right) U_{i}+\left(L_{i}\left(2 h_{i}^{*}+K_{V F i}\right) / K_{A i} K_{M i}\right) \ddot{\alpha}_{i}+\left(1 / K_{A i} K_{M i}\right) \times\left(L_{i} \dot{h}_{i}^{*}+\right. \\
& \left.+R_{i}\left(h_{i}^{*}+K_{V F i}\right)+K_{E i} K_{M i}\left(1-\left(\left(J_{i}+H_{i}^{*}\right) / J_{n i}\right)\right)\right) \dot{\alpha}_{i}+\left(R_{i} / K_{A i} K_{M i}\right) \times \\
& \times\left(n_{i}^{*}\left(1-\left(\left(J_{i}+H_{i}^{*}\right) / J_{n i}\right)\right)+M_{E i}^{*}+M_{Q F i}\right)+\left(L_{i} / K_{A i} K_{M i}\right) \times \\
& \times\left(\dot{n}_{i}^{*}\left(1-\left(\left(J_{i}+H_{i}^{*}\right) / J_{n i}\right)\right)+\dot{M}_{E i}^{*}\right) .
\end{aligned}
$$

Analysis of the components of equation (7) and expressions for $H_{i}, h_{i}, \dot{h}_{i}, M_{E i}$ and $\dot{M}_{E i}$ shows that realization of the control law (7) for each actuator does not present principal difficulties. The adaptive regulator included in the controller of actuator stabilizes its parameters at the nominal level.

To synthesize the simultaneousforce/position control law for actuator after stabilization its parameters at nominal level the optimal controller analytical design method [Naidu, 2002] will be used. The quadratic functional which simultaneously includes position error of the output shaft of actuator and error of the external moment developed by this actuator will be minimized. As a result (Filaretov \& Zuev, $2009)$ it will be possible to create the force/position regulators. Let the vector of the state variables will be $\mathrm{x}_{k i}=\left[\alpha_{i}, \dot{\alpha}_{i}, \mathrm{M}_{M i}\right]^{\mathrm{T}}(k=\overline{1,3})$ concerning to which the differential equations (7) of each manipulator's actuator in the Cauchy normal form will be 
Filaretov, V. \& Zuev, A.: An Advanced Approach to Automatic Machining of Com...

$$
\begin{aligned}
& \dot{x}_{1 i}=a_{12 i} x_{2 i}, \\
& \dot{x}_{2 i}=a_{23 i} x_{3 i}-a_{23 i} n_{i}^{*}, \\
& \dot{x}_{3 i}=a_{32 i} x_{2 i}+a_{33 i} x_{3 i}+b_{3 i} U_{i},
\end{aligned}
$$

where $a_{12 i}=\frac{1}{i_{R i}}, a_{23 i}=\frac{1}{J_{n i}}, a_{32 i}=-\frac{K_{M i} K_{E i}}{L_{i}}, a_{33 i}=-\frac{R_{i}}{L_{i}}, b_{3 i}=\frac{K_{A i} K_{M i}}{L_{i}}$.

Concerning errors $\varepsilon_{j i}=\mathrm{x}_{j i}-\mathrm{x}_{j i}^{d}$ and taking into account that $\dot{x}_{1 i}^{d}=a_{12 i} x_{2 i}^{d}$, $\dot{x}_{2 i}^{d}=a_{23 i} x_{3 i}^{d}-a_{23 i} n_{i}^{* d}, x_{3 i}^{d}=n_{i}^{* d}+\left(H_{i}^{*}+J_{i}\right) \dot{x}_{2 i}^{d}+\left(h_{i}^{*}+K_{V F i}\right) x_{2 i}^{d}+M_{E i}^{*}+M_{Q F i}, \dot{x}_{3 i}^{d}=\dot{n}_{i}^{* d}+\left(H_{i}^{*}+J_{i}\right) \ddot{x}_{2 i}^{d}+$ $+\left(2 h_{i}^{*}+K_{V F i}\right) \dot{x}_{2 i}^{d}+\dot{h}_{i}^{*} x_{2 i}^{d}+\dot{M}_{E i}^{*}$, system (8) can be rewritten in the form

$$
\begin{aligned}
& \dot{\varepsilon}_{1 i}=a_{12 i} \varepsilon_{2 i}, \\
& \dot{\varepsilon}_{2 i}=a_{23 i} \varepsilon_{3 i}+a_{23 i}\left(n_{i}^{* d}-n_{i}^{*}\right), \\
& \dot{\varepsilon}_{3 i}=a_{32 i} \varepsilon_{2 i}+a_{33 i} \varepsilon_{3 i}+b_{3 i} U_{i}+a_{32 i} x_{2 i}^{d}+a_{33 i} n_{i}^{* d}+ \\
& +a_{33 i}\left(H_{i}^{*}+J_{i}\right) \dot{x}_{2 i}^{d}+a_{33 i}\left(h_{i}^{*}+K_{V F i}\right) x_{2 i}^{d}+a_{33 i} M_{E i}^{*}+ \\
& +a_{33 i} M_{Q F i}-\dot{n}_{i}^{* d}-\left(H_{i}^{*}+J_{i}\right) \ddot{x}_{2 i}^{d}-\left(2 h_{i}^{*}+K_{V F i}\right) \dot{x}_{2 i}^{d}-\dot{h}_{i}^{*} x_{2 i}^{d}-\dot{M}_{E i}^{*},
\end{aligned}
$$

where $x_{i}^{d}(t) \in R^{3}$ is the vector of the desired values of phase coordinates; $n_{i}^{* d}=n_{i}^{d} / i_{R i}$; $n_{\mathrm{i}}^{d}$ is the desired external moment reduced to motor shaft which should be developed to $i$-th actuator for creation the desired resultant force exerted by CT.

In the matrix form system (9) becomes

$$
\dot{\varepsilon}_{i}=A_{i} \varepsilon_{i}+B_{i} U_{i}+\omega_{i}
$$

Where $A_{i}=\left[\begin{array}{ccc}0 & a_{12 i} & 0 \\ 0 & 0 & a_{23 i} \\ 0 & a_{32 i} & a_{33 i}\end{array}\right], B_{i}=\left[\begin{array}{c}0 \\ 0 \\ b_{3 i}\end{array}\right], \omega_{i}=\left[\begin{array}{c}0 \\ \omega_{2 i} \\ \omega_{3 i}\end{array}\right], \omega_{2 i}=a_{23 i}\left(n_{i}^{* d}-n_{i}^{*}\right)$,

$$
\begin{aligned}
& \omega_{3 i}=a_{32 i} x_{2 i}^{d}+a_{33 i} n_{i}^{* d}+a_{33 i}\left(H_{i}^{*}+J_{i}\right) \dot{x}_{2 i}^{d}+a_{33 i}\left(h_{i}^{*}+K_{V F i}\right) x_{2 i}^{d}+a_{33 i} M_{E i}^{*}+a_{33 i} M_{Q F i}- \\
& -\dot{n}_{i}^{* d}-\left(H_{i}^{*}+J_{i}\right) \ddot{x}_{2 i}^{d}-\left(2 h_{i}^{*}+K_{V F i}\right) \dot{x}_{2 i}^{d}-\dot{h}_{i}^{*} x_{2 i}^{d}-\dot{M}_{E i}^{*} .
\end{aligned}
$$

The control law for considered actuator (10) which minimizes the quadratic functional

$$
Y_{i}\left(U_{i}, \varepsilon_{i}\right)=\frac{1}{2} \int_{t_{0}}^{\widetilde{T}}\left(\varepsilon_{i}^{T} \Phi_{i} \varepsilon_{i}+U_{i}^{2}\right) d t,
$$

is formed in the form

$$
U_{i}(t)=-B_{i}^{T}\left(K_{i} \varepsilon_{i}+E_{i}\right),
$$


where $t_{0}, \widetilde{T}$ are initial and finite time of integration accordingly $(\widetilde{T} \rightarrow \infty) ; \Phi_{i} \in R^{3 \times 3}$ is certain positive semi-definite matrix of weight coefficients. $K_{i} \in R^{3 \times 3}$ is matrix whose elements are determined as a result the solution of the matrix equation of Riccati: $-\dot{K}_{i}=K_{i} A_{i}+A_{i}^{T} K_{i}-K_{i} B_{i} B_{i}^{T} K_{i}+\Phi_{i}, K_{i}(\widetilde{T})=0$.

Vector $E_{i} \in R^{3}$ is determined as a result of the solution of matrix equation: $E_{i}=\left(A_{i}^{T}-K_{i} B_{i} B_{i}^{T}\right)^{-1} K_{i} \omega_{i}$.

Taking this into account the control law (10) can be rewritten in the form

$$
U_{i}(t)=-B_{i}^{T}\left(K_{i} \varepsilon_{i}+\left(A_{i}^{T}-K_{i} B_{i} B_{i}^{T}\right)^{-1} K_{i} \omega_{i}\right)=U_{i}^{\lambda}+U_{i}^{e}=-D_{i}^{\lambda} \varepsilon_{i}+D_{i}^{e} \omega_{i},
$$

where $D_{i}^{\lambda}=B_{i}^{T} K_{i}, \quad D_{i}^{e}=-B_{i}^{T}\left(A_{i}^{T}-K_{i} B_{i} B_{i}^{T}\right)^{-1} K_{i} \in R^{3} \quad$ are vectors of amplification coefficients of corresponding communications.

Taking into consideration expression for $\mathrm{x}_{3 \mathrm{i}}^{d}$ and also that $\mathrm{x}_{1 \mathrm{i}}^{d}=\alpha_{\mathrm{i}}^{d}, \mathrm{x}_{2 \mathrm{i}}^{d}=\dot{\alpha}_{\mathrm{i}}^{d}$, $\dot{\mathrm{x}}_{2 \mathrm{i}}^{d}=\ddot{\alpha}_{\mathrm{i}}^{d}$ the law (13) for system (10) can be rewritten in the form:

$$
\begin{aligned}
& U_{i}(t)=-D_{1 i}^{\lambda} \alpha_{i}-\left\{D_{2 i}^{\lambda}-D_{2 i}^{e} a_{23 i}\left(h_{i}^{*}+K_{V F i}\right)\right\} \dot{\alpha}_{i}+D_{2 i}^{e} a_{23 i}\left(H_{i}^{*}+J_{i}\right) \ddot{\alpha}_{i}- \\
& -\left(D_{3 i}^{\lambda}+D_{2 i}^{e} a_{23 i}\right) K_{M i} I_{i}+D_{1 i}^{\lambda} \alpha_{i}^{d}+\left\{D_{2 i}^{\lambda}+D_{3 i}^{e}\left(a_{32 i}-\dot{h}_{i}^{*}\right)+\left(D_{3 i}^{\lambda}+D_{3 i}^{e} a_{33 i}\right) \times\right. \\
& \left.\times\left(h_{i}^{*}+K_{V F i}\right)\right\} \dot{\alpha}_{i}^{d}+\left\{\left(D_{3 i}^{\lambda}+D_{3 i}^{e} a_{33 i}\right)\left(H_{i}^{*}+J_{i}\right)-D_{3 i}^{e}\left(2 h_{i}^{*}+K_{V F i}\right)\right\} \ddot{\alpha}_{i}^{d}+ \\
& +\left\{D_{3 i}^{\lambda}+D_{2 i}^{e} a_{23 i}+D_{3 i}^{e} a_{33 i}\right\} n_{i}^{* d}-D_{3 i}^{e} \dot{n}_{i}^{*}+\left\{D_{3 i}^{\lambda}+D_{2 i}^{e} a_{23 i}+\right. \\
& \left.+D_{3 i}^{e} a_{33 i}\right\}\left(M_{E i}^{*}+M_{Q F i}\right),
\end{aligned}
$$

where $D_{1 i}^{\lambda}, D_{2 i}^{\lambda}, D_{3 i}^{\lambda}, D_{2 i}^{e}, D_{3 i}^{e}$ are corresponding elements of vectors $D_{i}^{\lambda}, D_{i}^{e}$. It should be noted that in expression (14) the connections which contain the coordinate $\dddot{\alpha}_{i}^{d}$ are excluded since the results of the conducted investigations (Filaretov, 2008) showed the small influence of this connection on the precision of the synthesized system. The structure of the control law (14) is shown on Fig. 9.

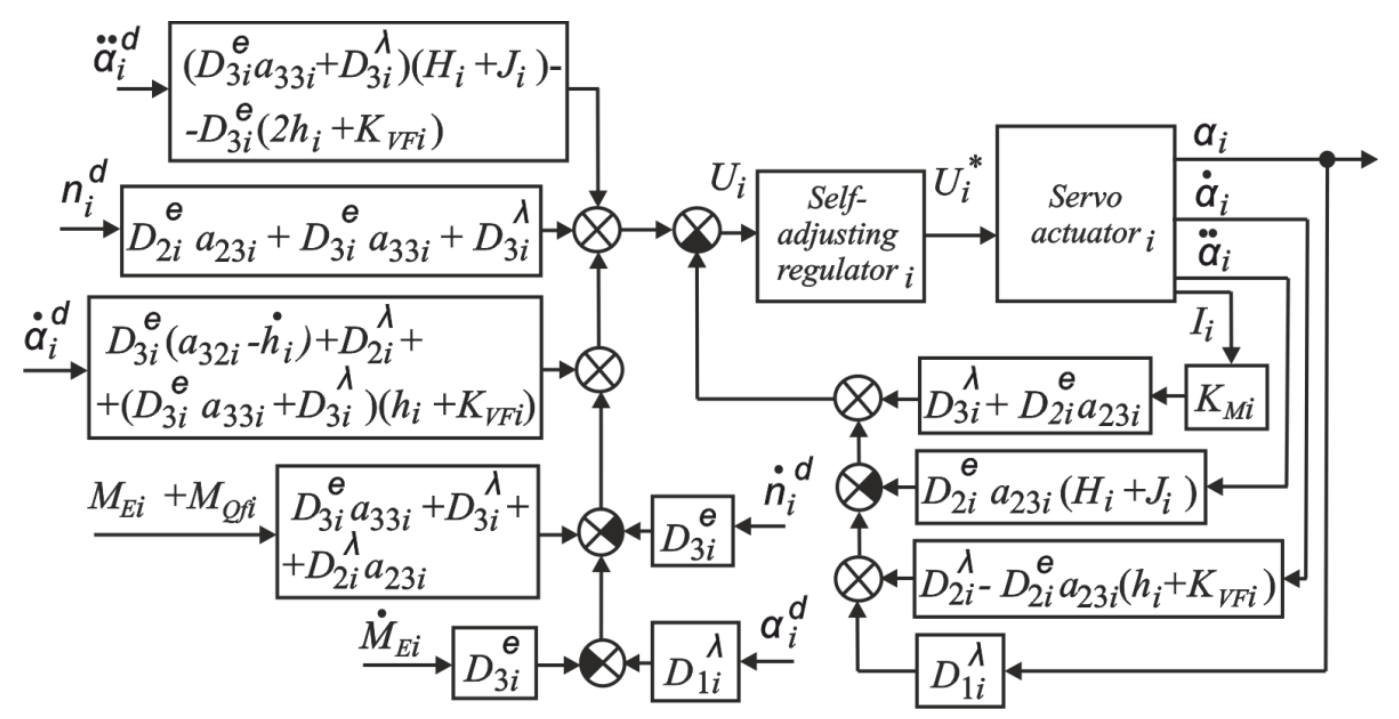

Fig. 9. Block diagram of the $i$-th actuators with the force/position regulator 
To realise the specified law it is necessary to enter feedback on coordinates of actuators: $\alpha_{i}, \dot{\alpha}_{i}, \ddot{\alpha}_{i}, I_{i}$ and also connections on the desirable values: $\alpha_{i}^{d}, \dot{\alpha}_{i}^{d}, \ddot{\alpha}_{i}^{d}, n_{i}^{*_{d}}$, $\dot{n}_{i}^{* d}$. Laws of change $\alpha_{i}^{d}=q_{i}^{d}$ are defined from the decision of a return problem of kinematics for concrete manipulators. If laws of change $\alpha_{i}^{d}$ and $n_{i}^{* d}$ are previously known then to obtain the laws of change of coordinates $\dot{\alpha}_{i}^{d}, \ddot{\alpha}_{i}^{d}, \dot{n}_{i}^{* d}$ also does not represent difficulties. All these signals are formed before the start of manipulator's work.

Note that the control laws (7) and (14) contain the components proportional to angular accelerations of rotors all engines of the manipulator. These components are present always when sizes of inductances of electric motor rotor circuits $L_{i}$ of used engines are considerable. However in many modern electric motors these inductances are very small and it is possible simply to disregard them. In this case the specified components in equations (7) and (14) vanish.

Thus the obtained law of control (14) allows simultaneously accurately to control of both the position of the output shaft of actuators and external moment created by this actuator since ensures the minimization of functional (11), which contains the position error and moment error.

To realize the force/position control it is necessary formation in real time the program values of the external moments $n_{i}^{d}$ for each DOF of MM which overall create of required force $F^{e x}=\left[f^{e x}: n^{e x}\right]^{T}$ (see Fig. 8) to PCM part.For this it is necessary to connect the moments in DOFs with the generalised force on CT. Generally for this purpose relation of the external torque vector with generalized force through the Jacobian matrix of manipulator is used. But in this case, there are some difficulties related with compound computing for complex manipulators. Therefore, we propose the recurrence equations for calculation of the desired external torque for manipulators with any kinematics in real time. To describe manipulator's mechanism, it is possible to use the Denavit-Hartenberg modified notation (Poul, 1981), which considers four parameters $\hat{\theta}_{i}, \widehat{\alpha}_{i}, \widehat{a}_{i}$, and $\widehat{d}_{i}$, as shown in Fig. 10.

In the mentioned notation, the reference system corresponding to link $i$ is located on joint $i$, and the $Z$-axis is located on the axis in the same node, which connects links $i-1$ and $i$. The reference system $i+1$ is related to the $i$ reference system by means of the rotation matrix ${ }^{i} R_{i+1}$ and the position vector ${ }^{i} p_{o_{i}, o_{i+1}}$ :

$$
{ }^{i} R_{i+1}=\left[\begin{array}{ccc}
\cos \hat{\theta}_{i+1} & -\sin \hat{\theta}_{i+1} & 0 \\
\cos \hat{\alpha}_{i+1} \sin \hat{\theta}_{i+1} & \cos \hat{\alpha}_{i+1} \cos \hat{\theta}_{i+1} & -\sin \hat{\alpha}_{i+1} \\
\sin \hat{\alpha}_{i+1} \sin \hat{\theta}_{i+1} & \sin \hat{\alpha}_{i+1} \cos \hat{\theta}_{i+1} & \cos \hat{\alpha}_{i+1}
\end{array}\right],{ }^{i} p_{O_{i}, O_{i+1}}=\left[\begin{array}{c}
\hat{a}_{i+1} \\
\hat{d}_{i+1} \sin \widehat{\alpha}_{i+1} \\
-\hat{d}_{i+1} \cos \hat{\alpha}_{i+1}
\end{array}\right] .
$$

The force of reaction $\hat{f}_{i}\left(\hat{f}_{i} \in R^{3}\right)$ or the moment of force $\widehat{n}_{i}\left(\hat{n}_{i} \in R^{3}\right)$ (according to type of joint) will be acting in joint $i$ of manipulator when its end-effector will exerts some generalized force on PCM part. 


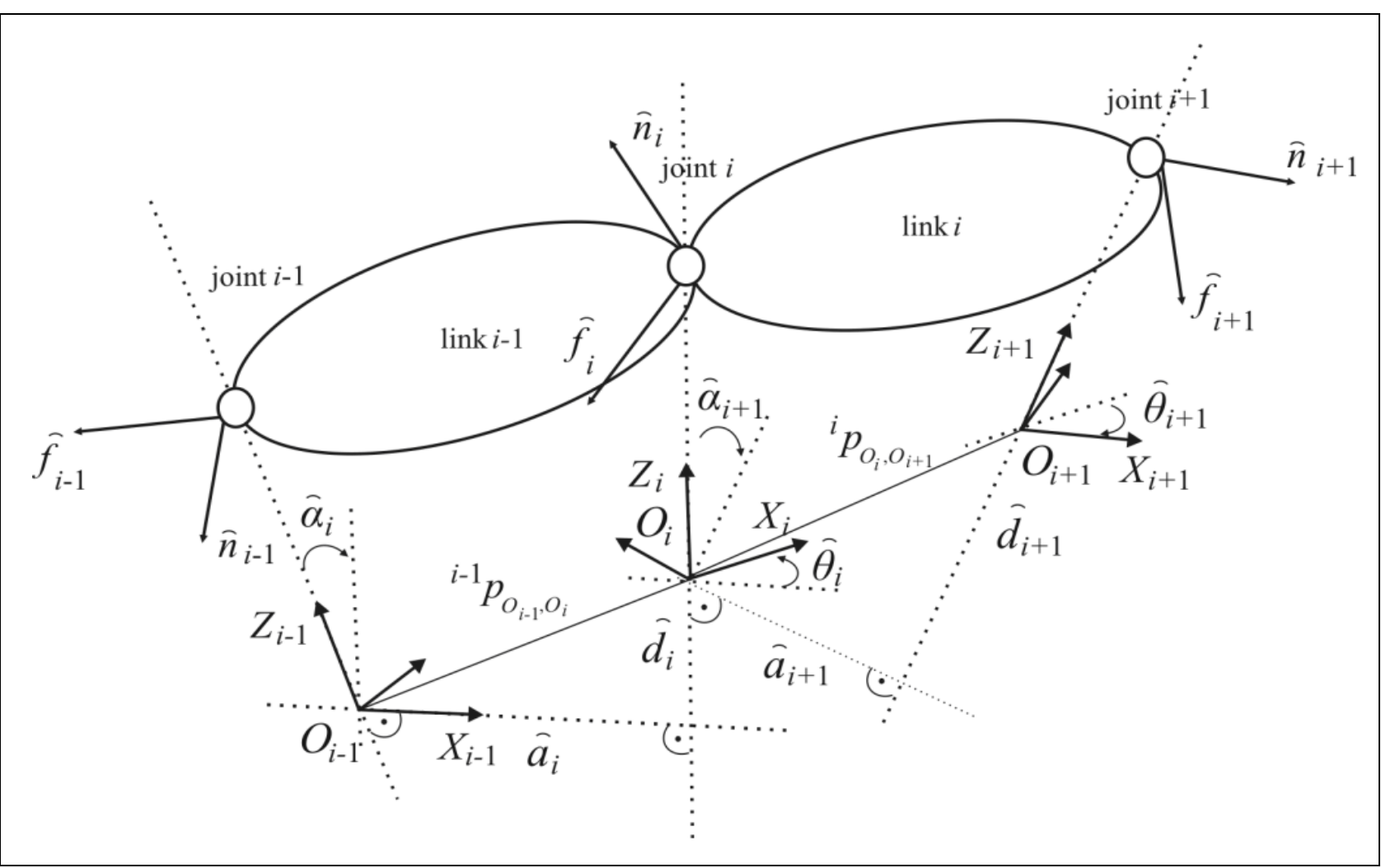

Fig. 10. Systems of coordinates and the main vectors of the manipulator's link

Therefore, the recurrence allowing calculation of desirable (program) values of external loading torques $n_{i}^{d}$ in each joint of any manipulator which provides force and moment by end-effector on PCM part will be

$$
\begin{aligned}
& \widehat{f}_{i}={ }^{i} R_{i+1} \widehat{f}_{i+1}, \quad \widehat{f}_{i+1}=f^{e x}, \quad(i=\overline{n, 1}), \\
& \widehat{n}_{i}={ }^{i} R_{i+1} \widehat{n}_{i+1}+{ }^{i} p_{O_{i}, O_{i+1}} \times\left({ }^{i} R_{i+1} \hat{f}_{i+1}\right), \quad \hat{n}_{i+1}=n^{e x}, \\
& n_{i}^{d}=e_{i}^{\mathrm{T}}\left(\widehat{f}_{i} \delta_{i}+\bar{n}_{i} \bar{\delta}_{i}\right),
\end{aligned}
$$

where $\delta_{i}=1$ if joint $i$ is progressive and $\delta_{i}=0$ if it is rotative; $\bar{\delta}_{i}=1-\delta_{i} ; e_{i}^{\mathrm{T}}=(0,0,1)$ is a unit vector connected and directed along the $Z_{i}$ axis; $f^{e x}, n^{e x}$ are the force and moment (parts of generalized force) exerted be CT on PCM part.

Thereby, the recurrences (15) allow calculation on the values $n_{i}^{d}$ in real time for any multilink manipulators. The executed synthesis of simultanious force/position control system of MM without decrease in rigidity of MM and use of force sensors allowed to solve theoretically a problem of possible machining of difficult freely deformable PCM parts.

Thus, offered approach to formation of trajectories of movement CT with SVS use at deformation of separate segments of PCM parts after their fixing allows to carry out automatically machining of them at any deformation in the course of processing. 
Filaretov, V. \& Zuev, A.: An Advanced Approach to Automatic Machining of Com...

\section{Results of Experimental Studies of Proposed Approach to Machining of PCM Parts by Means of MM With SVS}

To confirm the efficiency of the chosen strategy and approaches to machining of nonrigid PCM parts by means of MM with SVS were carried out experimental researches of efficiency of processing of details with use of information on spatial cutting line received from SVS. In particular, the accuracy of determination of coordinates of the points forming line marked on part, by means of this SVS and also the accuracy of tracking of the received trajectory by CT was investigated.

At experimental researches, the DENSO VS-6556G B/RC 7M manipulator and the stereo camera Bumblebee ${ }^{\circledR} 2$ 1024x768 which mounted near with end-effector were used. This of experimental installation is shown in fig. 11a.

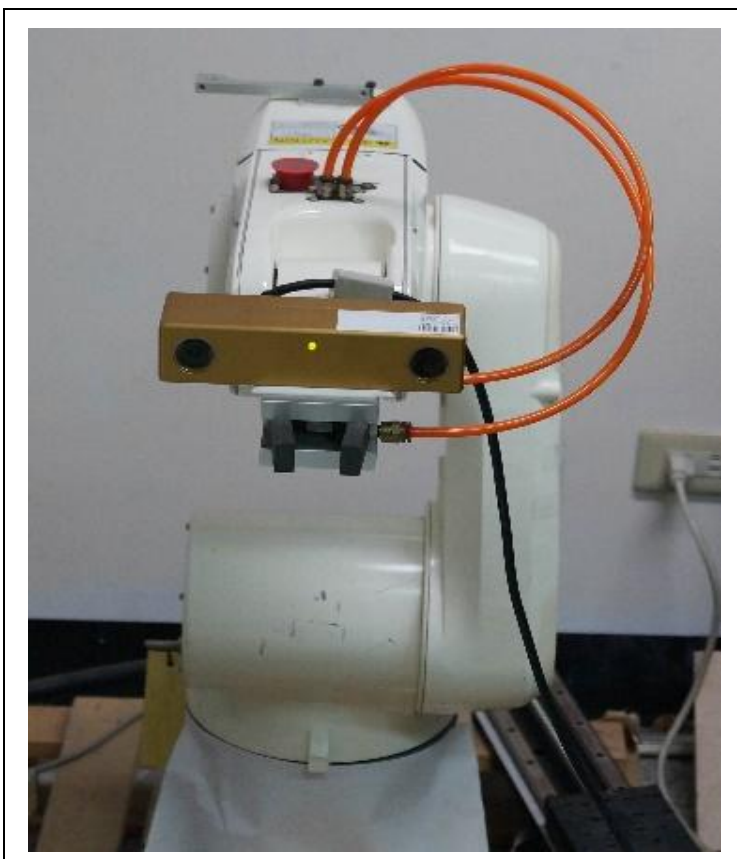

a)

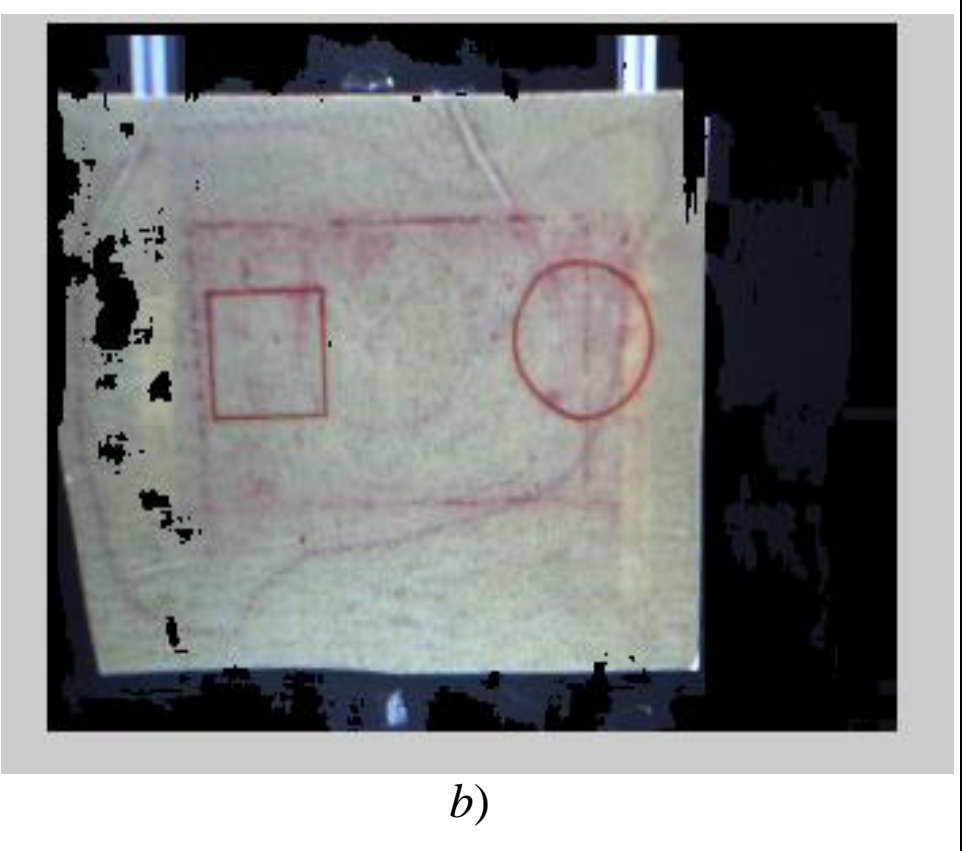

Fig. 11. General view of experimental installation

Before carrying out of experiment in working zone of MM arbitrarily at some distance PCM part with previously marked contours in the form of a square with sides of $72 \mathrm{~mm}$ and circles with radius of $3,5 \mathrm{~mm}$ (see fig. 11b) was established. Preliminary calibration of SVS was carried out by means of a Camera Calibration Toolbox of MATLAB.

In the course of carrying out experiment consecutive recognition of the marked trajectories, definition of their spatial position and orientation in coordinate system of SVS was carried out. Experiments showed that errors of determination of lengths of the side of square and radius of circle didn't exceed values of $0,3 \mathrm{~mm}$ and $0,42 \mathrm{~mm}$, respectively. 
In experiment imitation of automatic machining by means of CT (the simulator of a laser cutting head) flat part on the revealed contours was made. The error of working off of CT of both trajectories revealed by means of SVS didn't exceed $0.6 \mathrm{~mm}$.

As a whole, the provided experimental researches completely confirmed the efficiency of offered approaches and algorithms, and also operability of the technical solutions which have provided high precision of processing of flat PCM parts by means of MM with SVS.

\section{Conclusion}

The conducted researches and experiments confirmed possibility of highprecision automatic machining of PCM parts by means of the MM equipped with SVS without use of expensive individual equipment for their rigid fixing before processing.

For effective realization of the offered approaches it is necessary to use highspeed algorithms of exact recognition and determination of spatial coordinates of the points forming marked trajectories of cutting on processed parts, especially when these parts have a difficult spatial surface, and the marked trajectories aren't accurately recognized on processed surface.

\section{References}

Comport, A., Marchand, E., Pressigout, M., Chaumette, F. (2006). Real-time markerless tracking for augmented reality: the virtual visual servoing framework. IEEE Transactions on Visualization and Computer Graphics, vol.12, no.4, pp. $615-628$

Craig, J.(2003). Introduction to robotics: mechanics and control. Prentice Hall. Fairhurst, M. (1988).Computer Vision for Robotic Systems: An Introduction. Prentice Hall

Filaretov, V. \& Zuev, A. (2008). The combined force/position control systems for manipulators.Proc. of the 9th Biennial ASME Conference on Engineering Systems, Design and Analysis, Haifa, Israel, pp. 1-7

Filaretov, V. (1993). A Synthesis of Adaptive Control Systems For Industrial Robots. Proc.Of Japan Int. Electronic Manufacturing Technology Symp, Kanazawa, Japan, pp. 168 - 171.Filaretov, V. \&Vukobratovic, M. (1995).Synthesis of Adaptive Robot Control Systems for Simplified Forms of Driving Torques.Mechatronics, vol. 5, no 1, pp. $41-59$

Filaretov, V. Yukhimets, D., Konoplin, A. (2012). Synthesis method of automatic control of regimes of motion of end-effector of manipulator on spatial trajectories. Mechatronics, automation and control, no.6, pp. 47-54.(In Russian)

Filaretov, V., Zuev, A., Gubankov, A. (2001).The development of robotic system for machining of nonrigit spatial composite parts of helicopters.IzvestyaVuzov. Mashinostroenie, pp.67-75.(In Russian)

Forsyth, D. \& Ponce, J. (2011).Computer Vision: A Modern Approach. Prentice Hall. 
Filaretov, V. \& Zuev, A.: An Advanced Approach to Automatic Machining of Com...

Grammatikopoulos, L., Karras, G., Petsa, E. (2004). Camera calibration combining images with two vanishing points.International Archives of the Photogrammetry, Remote Sensing \& Spatial Information Sciences, vol. 35, no.5, pp.99-104

Gruen, A.\& Huang, T. (2001). Calibration and Orientation of Cameras in Computer Vision.Springer

Klank, U., Pangercic, D., Rusu, R. and Beetz, M. (2009).Real-time CAD model matching for mobile manipulation and grasping. Proc. of the 9th IEEE-RAS International Conference on Humanoid Robots, pp. 290 - 296

Le Duc, H, Lin, J. (2012). Combining stereo vision and fuzzy image based visual servoing for autonomous object grasping using a 6-DOF manipulator. Proc. of the 2012 IEEE International Conference on Robotics and Biomimetics, pp. 1703 - 1708.

Madison, J. (1996). CNC Machining Handbook: Basic Theory, Production Data, and Machining Procedures. Industrial Press, Inc

Marshall, G.\&Stutz, G. (2011) Handbook of Optical and Laser Scanning, Second Edition (Optical Science and Engineering). CRC Press

Mezouar, Y. \&Chaumette, F. (2002).Path Planning for Robust Image-based Control.IEEE Trans. on Robotics and Automation, vol. 18, no. 4, pp. 534-549

Naidu, S. (2002). Optimal Control Systems (Electrical Engineering Handbook).CRC. Perks, A. (2006). Advanced vision guided robotics provide 'future-proof' flexible automation. Assembly Automation, vol.26, no.3, p. 216-217

Poul, R. (1981). Robot manipulators: mathematics, programming and control. Cambridge: MIT

Puppo, E. (1998), Variable resolution triangulations. Computational Geometry, vol. 11, pp. $219-238$

Sebastian, J., Pari, L., Angle, L. \&Traslosheros, A. (2009).Uncalibrated visual servoing using the fundamental matrix. Robotics and Autonomous Systems, vol. 57(1), pp. 1-10

Shapiro, L. (2001). Computer Vision.Prentice Hall

Youdong, L. (2000). A new definition and calculation for the average normal to a polygon.Sciense in China (Series E), vol. 43, no.5

Zuev, A. \& Filaretov, V. (2009). Features of Designing Combined Force/Position Manipulator Control Systems. Journal of Computer and Systems Sciences International, vol. 48, no. 1, pp.146-154

ZuWhan, K. (2006). Geometry of Vanishing Points and its Application to External Calibration and Realtime Pose Estimation. Research Reports, Institute of Transportation Studies (UCB), UC Berkeley

Chaumette, F. \& Hutchinson, S. (2006). Visual servo control. Part I. Basic approaches. IEEE Robotics and Automation Magazine, vol.13, no.4, pp.82-90

Chaumette, F. \& Hutchinson, S. (2007). Visual servo control. Part II. Basic approaches.IEEE Robotics and Automation Magazine, vol.15, no.1, pp.109-118 